\title{
Assessment of autonomous phase unwrapping of isochromatic phase maps in digital photoelasticity
}

\author{
M RAMJI, E NITHILA, K DEVVRATH and K RAMESH* \\ Solid Mechanics Group, Department of Applied Mechanics, Indian Institute \\ Technology Madras, Chennai 560036 \\ e-mail: kramesh@iitm.ac.in
}

MS received 9 June 2006; accepted 24 January 2007

\begin{abstract}
Photoelasticity is the only whole-field experimental technique which can analyse both 2-D and 3-D elasticity problems. In digital photoelasticity one gets two phase maps, one corresponding to principal stress direction (isoclinic) and the other corresponding to principal stress difference (isochromatic). The phase maps for both isoclinics and isochromatics are to be unwrapped differently for obtaining continuous phase values. Autonomous phase unwrapping is one of the challenging issues and this paper focuses on recent advances on isochromatic phase map unwrapping. A comparative study of different autonomous phase unwrapping algorithm is done by solving a bench mark problem and a stress frozen slice with cut-outs. The need for domain delimiting in addition to domain masking is brought out while using autonomous phase unwrapping algorithms.
\end{abstract}

Keywords. Phase map; unwrapping; digital photoelasticity; isochromatic.

\section{Introduction}

Photoelasticity is an optical technique which yields the information of constant principal stress difference (isochromatics) and constant principal stress direction (isoclinics) in the form of fringe contours. The technique is unique in the sense that it is the only whole field technique which can analyse the interior of 3-D models. Though photoelasticity is a whole-field technique, in the early days of its development isoclinic and isochromatic data were obtained only at the fringe contours. With the advent of digital computers recording of images as intensity data became easier and a separate branch of photoelasticity namely 'digital photoelasticity' came into existence Ramesh (2000). In digital photoelasticity one gets isoclinic $(\theta)$ and isochromatic $(N)$ data for every point in the domain. The various techniques of digital photoelasticity can be broadly classified as polarization stepping, phase shifting, load stepping and Fourier transform approach Ramesh (2000). In most of these techniques, a few images whose number depends on the particular choice of the algorithm are recorded using a CCD camera by altering the angular positions of the optical elements. By appropriately processing

\footnotetext{
${ }^{*}$ For correspondence
} 
the intensity data thus recorded, the wrapped values of $\theta$ and $N$ are obtained. The wrapped values are represented as an image called phase map, which is different from the conventional fringe patterns of photoelasticity. The phase maps have to be unwrapped for getting the continuous phase values. Among the various digital photoelastic techniques, phase shifting technique (PST) is the most widely used for isochromatic phase map evaluation due to its simplicity and accuracy. Of all the PST methods, six-step method stands out as it effectively accounts for the quarter-wave plate mismatch error (Ramesh 2000; Ajovalasit et al 1998) and the role of background intensity (Sai Prasad \& Ramesh 2003). In this paper, unwrapping of only isochromatic phase maps is dealt with.

Phase unwrapping is widely used in other fields of interferometry such as holography, speckle and Moiré deflectometry. One of the main problems in all these techniques is the presence of noise in the phase map. This needs to be handled appropriately while unwrapping the phase. Itoh (1982) proposed simple 1-D and 2-D sequential scanning phase unwrapping algorithms. Ghigilia et al (1987) proposed a method in which inconsistencies in the 2-D phase map are uncovered by using cellular automata method. Later, Goldstein et al (1988) showed that the inconsistencies or local error can propagate to form global error that affect the entire image. To avoid this, branch-cuts are placed to prevent error propagation. Huntley (1989) devised an algorithm that combines noise immunity with computational efficiency.

Bone (1991) was the first to adopt a quality measure to guide the unwrapping process. He proposed a method based on the second difference of the locally unwrapped phase in which the conflictive points are selected by comparison of the value of the second difference with a fixed threshold. This method helps in detecting the phase discontinuities that produce integer fringe shifts. Quiroga et al (1995) enhanced Bone's technique by applying an adaptive threshold instead of a fixed one and also the second difference of the ideally unwrapped phase as a selection criterion for the pixels to be processed. Lim et al (1995) extended these techniques to a true quality-guided path following by allowing the threshold to increase as the unwrapping progressed. This had the effect of unwrapping the highest-quality pixels first, followed by lower quality pixels until all the pixels were unwrapped. Xu \& Cumming (1996) improved it by growing the highest quality regions independently of one another and then join them in a post processing step. An excellent summary of phase unwrapping techniques applied to magnetic resonance imaging (MRI) images, interferometric synthetic aperture radar (IFSAR) data is provided by Ghiglia \& Pritt (1998).

In digital photoelasticity, one of the key issues that needs to be addressed while unwrapping is the ambiguity in the sign of the fractional retardation more than the noise as in other interferometric techniques. Due to difficulties in not obtaining $\theta$ corresponding to either $\sigma_{1}$ or $\sigma_{2}$ uniformly over the complete domain Ramesh (2000), the sign of the fractional retardation is not known precisely. This leads to a situation wherein the fractional fringe order may increase or decrease in the direction of increase in total fringe order. Due to this, the phase map will have distinct zones. Unwrapping procedure adopted for one zone will not be applicable to the other zone. This requires the identification of the boundary of these zones and appropriate processing of data contained in these zones. Several ambiguity removal algorithms such as use of fuzzy sets by Wang \& Paterson (1995), Ramesh \& Mangal (1999) interactive approach, load stepping approach by Ekman \& Nurse (1998), Sai Prasad et al (2004) image-processing approach and Prashant et al (2004) theta checking approach are available to address the ambiguity issue. Prashant et al (2004) have identified different class of ambiguous boundary zones and suggested which of the ambiguity removal algorithms needs to be applied. 
Use of ambiguity removal algorithms followed by unwrapping has been emphasized by Ramesh (2000) and Sai Prasad et al (2004) for effective phase unwrapping in photoelasticity. Use of simple row-wise scanning or column-wise scanning for unwrapping and their respective choice in the presence of cut-outs have also been brought out Ramesh (2000). Extending the idea of phase unwrapping in other fields of interferometry, Asundi \& Wenson (1998) proposed a fast phase unwrapping algorithm for processing isochromatic phase maps in digital photoelasticity based on gray scale masking and flood-fill approach so that it unwraps phase from an area with higher reliability to the one with lower reliability. Quiroga \& Gonzalez (2000) presented a regularization algorithm for processing photoelastic fringe pattern using a modified cost functional approach. Servin \& Quiroga (2001) used the contrast of the isochromatic fringes as a measure of quality to unwrap ischromatic fringe order from a single dark field image recorded. In order to account for choice of specific unwrapping schemes for different regions, Sai Prasad et al (2002) developed a flexible phase unwrapping algorithm using tiling. Recently, Jones \& Wang (2003) have compared the Goldstein's branch-cut algorithm for isochromatic phase unwrapping with their newly proposed fringe combination matching methodology. Siegmann et al (2005) have used a quality-guided algorithm involving phase derivative variance for isochromatic phase map unwrapping of a complex aircraft component. Quality-guided path following algorithm and Goldstein's branch-cut algorithm have autonomous unwrapping capability. In view of this, it is desirable to use these methodologies for phase unwrapping in digital photoelasticity.

Although use of branch-cut and quality-guided path following are in use in other fields of interferometry, it is only at an initial stage as far as photoelasticity is concerned. In order to fully exploit the advantage of these new developments, in this paper, initially the basic methodology of various autonomous phase unwrapping algorithms such as Goldstein branchcut and various quality-guided path follower methods (Ghiglia \& Pritt 1998) are briefly presented. The implementation of the various algorithms discussed is validated for the problem of a disk under diametral compression. Unwrapping schemes can become erroneous when there are geometric discontinuities in the model. A new methodology is proposed to effectively unwrap the isochromatic phase maps of models having geometric discontinuities by adopting the concept of domain masking. In domain masking, certain unwanted regions (in this case cut-outs) are masked and the unwrapping scheme will omit such regions thereby ensuring proper unwrapping. The advantage of the proposed methodology is brought out by solving the problem of a stress frozen slice with cut-outs. The example problem chosen also requires the use of domain delimiting for effective phase unwrapping.

\section{Phase unwrapping - An introduction}

Phase unwrapping is a non-linear process which involves adding integer multiples of $2 \pi$ to the wrapped phase values in order to get continuous phase data. This process is normally carried out by successive comparisons of neighbouring pixels. It is generally assumed that the phase map is slowly varying: each pixel varies less with respect to its adjacent neighbour except at wrapped arctangent jump. On implementing the arc-tangent function, the actual phase function $\varphi[n]$ is wrapped into the interval $[0,2 \pi]$ by the following operation:

$$
W\{\varphi[n]\}=\delta[n]=\varphi[n]+2 \pi k[n],
$$

where $W$ is the wrapping operator, $n$ is an integer number, $\delta[n]$ is the wrapped phase and $k[n]$ is an integer function that forces $\delta$ to lie between 0 to $2 \pi$. One of the important concerns 
in unwrapping algorithms is whether the $2 \pi$ jump in the wrapped phase map is induced by an arctangent function or is it due to noise. Phase unwrapping algorithms can be broadly classified as follows:

(i) Path-following algorithms

(ii) Region algorithms

(iii) Global algorithms.

The path following algorithms proceeds in simple/complicated paths. Simple path following algorithms may be row-wise, column-wise, diagonal-wise scanning, etc. If the path is determined by quality of wrapped phase values pixel-wise then unwrapping path is complicated. The local weights (quality/branch-cut) guide the selection of the unwrapping path. The noisiest pixels get unwrapped at last to prevent the error propagation.

The region algorithms can be sub-classified into two groups: the tile-based group and the region-based group. The tile-based algorithms divide the image into a grid of smaller areas that are unwrapped individually. The unwrapped tiles are then combined together and unwrapped with respect to each other. The region-based group algorithms divide the image into homogenous regions within which the phase is continuous. This is achieved by comparing a pixel and its neighbour. If the phase difference is within a predetermined value, then the pixel and its neighbour are allocated to the same region; otherwise, they belong to different regions. After that, the regions are shifted with respect to each other to eliminate the phase discontinuities.

The global algorithm formulates the unwrapping algorithm in terms of minimization of a global function. These are mathematical function's like Green's, Helmholtz, etc. The main idea of this approach is to get the unwrapped phase values as solutions which minimizes error norm when substituted to the functions along with proper boundary values. In comparison to other interferometry techniques like speckle, though noise is not a major concern in digital photoelasticity it is desirable to use an algorithm which is immune to the presence of noise in photoelasticity.

\section{Methodology of various autonomous algorithms}

Phase unwrapping algorithms must include procedures to handle problems such as noise or abrupt fringe breaks to achieve a final error-free continuous phase map. In general, points that present these types of problems must not be processed but inferred from the continuous phase map. However, it is difficult to identify all the conflictive points. In such cases the points that are suspected to be conflictive must be, at the least, the last to be unwrapped to avoid error propagation across the continuous phase map.

\subsection{Goldstein's branch-cut algorithm}

This algorithm identifies what are known as 'residues' in the wrapped phase data and 'balances' them with branch-cuts. Choice of the branch-cut has a great influence on the quality of the final unwrapped phase. Residues are inconsistent points caused due to phase discontinuities/noise that are present in the phase map. Residues can be positive or negative. The discontinuities are assumed to lie on approximate shortest paths between positive and negative residues. An unique, unwrapped solution results from the selection of a wrapped-gradient integration path that avoids these discontinuities. The algorithm has three steps of identification of residues, generation of branch-cuts followed by a flood fill algorithm for phase unwrapping. These are discussed next. 


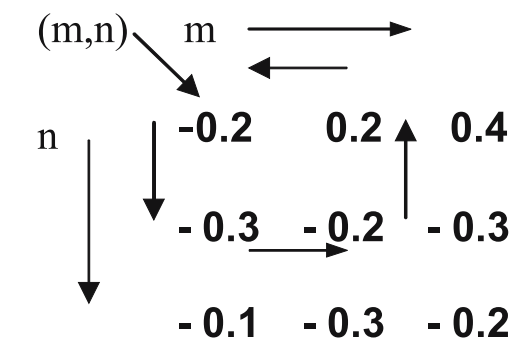

(a) $\quad q=0$; no residue

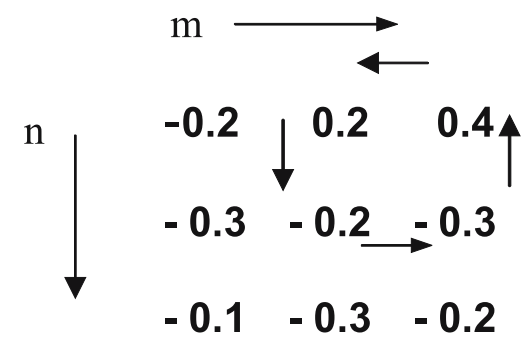

(b) $q=-1$; negative residue

Figure 1. A small portion of the typical array of wrapped phase values. The samples represent the phase in cycles and are multiplied by $2 \pi$ to obtain the wrapped phase in radians.

3.1a Residues: The phase map has gray scale intensity values in the range $0-255$ that is scaled to $0-1$ and read in an array. The $2 \times 2$ sample path integrals is the smallest closed loop that can be defined in the sampled image. This small loop helps in localizing the source of each phase inconsistency. Summation of the gradient of the wrapped phase (figure 1) defined as $q$ is obtained as follows:

$$
q=\sum_{i=1}^{4} \Delta_{i}
$$

where,

$$
\begin{aligned}
& \Delta_{1}=\{\delta(m, n+1)-\delta(m, n)\} \\
& \Delta_{2}=\{\delta(m+1, n+1)-\delta(m, n+1)\} \\
& \Delta_{3}=\{\delta(m+1, n)-\delta(m+1, n+1)\} \\
& \Delta_{4}=\{\delta(m, n)-\delta(m+1, n)\},
\end{aligned}
$$

when $q$ is non zero, inconsistency exists and is considered as a residue. It can be positive or negative and it can have values $0,+1$ or -1 . It is not possible for the $2 \times 2$ path consistency check to determine if an inconsistency is due to aliasing or noise. This is because the consistency check is a local test, whereas a phase dislocation is a regional phenomenon. It is assumed that the data are adequately sampled; to satisfy Nyquist criterion, i.e. there cannot be a jump of more than one-half cycle from point-to-point.

3.1b Branch-cut generation: In Goldstein et al (1988) algorithm, line segments (branches) are drawn between positive and negative residues in a systematic way to function as barriers during the path integration that cannot be crossed. Algorithm begins by scanning the phase map until a residue is found. Then a tile of $3 \times 3$ is placed around the residue and is searched for another residue. When a residue of opposite sign is found, a branch-cut is placed between the two and the scanning continues for finding another residue. But if residue of same sign appears, the centre of the box is moved to the new residue location and the search continues until either an opposite residue is found or no new residues can be found within the tile. When no residue is found, the tile size is increased by two $(5 \times 5)$ and the search continues from the current starting residue. Likewise, the algorithm scans over the entire phase map 
for unbalanced residues and balances them by placing branch-cuts. Sometimes when border pixels are encountered during the box search, residues can also be balanced by connecting them with the image border. The cuts are generated by a nearest-neighbour method that attempts to minimize the sum of the cut lengths. The Goldstein's algorithm is effective at generating optimal branch-cuts and is extremely fast (Goldstein et al 1988).

3.1c Unwrapping algorithm: At first, an initial seed value is given at a known location within the model domain. The four neighbouring pixels are thus unwrapped and the algorithm is implemented in a sense that it goes around the location of branch-cut pixels. The unwrapping of the branch-cut pixels is executed in the end by using the unwrapped value of its adjacent pixels. In this way the error is prohibited from propagating to other zones of the phase map.

\subsection{Quality-guided path follower algorithm}

In recent years, several statistical measures have been proposed that decides on the reliability of the phase value of a pixel. The application of these statistical measures to the whole domain results in a map called quality map. These are arrays of values that indicate the quality or goodness of the phase data. The pixels in the phase map are graded depending on its quality where ' 1 ' refers to the highest quality and ' 0 ' denotes the lowest quality. The algorithms which use these maps as a source of reliability information for unwrapping are known as quality-guided path followers. It is essentially a region growing approach that identifies the highest quality pixels, unwraps them first, and avoids the low quality pixels until the end of the unwrapping procedure. There are mainly four quality maps in use and they influence the performance of the unwrapping algorithms in varying degrees. They are:

(i) Correlation map

(ii) Pseudo-correlation map

(iii) Phase derivative variance map

(iv) Maximum phase gradient map.

The best quality map applicable for a problem is decided by trial and error basis.

3.2a Correlation map: The correlation map finds extensive usage in IFSAR data analysis. Certain correlation coefficients are directly extracted from IFSAR data.

3.2b Pseudo-correlation map: This map estimates correlation coefficients between the phase data. It is defined as

$$
\frac{\sqrt{\left(\sum \cos \delta_{i, j}\right)^{2}+\left(\sum \sin \delta_{i, j}\right)^{2}}}{k^{2}}
$$

where the sums are evaluated in the $k \times k$ neighbourhood of each center pixel $(m, n)$ and $\delta_{i, j}$ are the wrapped values.

3.2c Phase derivative variance map: This quality map gives the measure of the statistical variance of the phase derivatives. Since it involves derivatives, this quality map is more suitable for phase data with steep gradients. It is defined as

$$
\frac{\sqrt{\sum\left(\Delta_{i, j}^{x}-\bar{\Delta}_{m, n}^{x}\right)^{2}}+\sqrt{\sum\left(\Delta_{i, j}^{y}-\bar{\Delta}_{m, n}^{y}\right)^{2}}}{k^{2}}
$$


where for each sum the indices $(i, j)$ range over the $k \times k$ neighbourhood of each center pixel $(m, n)$ and $\Delta_{i, j}^{x}$ and $\Delta_{i, j}^{y}$ are the partial derivatives of the phase (i.e. the wrapped phase differences). The terms $\bar{\Delta}_{m, n}^{x}$ and $\bar{\Delta}_{m, n}^{y}$ are the averages of these partial derivatives in the $k \times k$ windows. Equation (5) is a root-mean-square measure of the variances of the partial derivatives in the $x$ and $y$ directions. The phase derivative variance map differs from the correlation and pseudo-correlation map in a way that it indicates the 'badness' rather than the goodness of the phase data. The quality value is negated consequently to represent goodness.

3.2d Maximum phase gradient map: This map measures the magnitude of the largest phase gradient (i.e. partial derivative of wrapped phase difference) in the $k \times k$ neighbourhood of each pixel. It is defined to be the greater of the two values

$$
\max \left\{\left|\Delta_{i, j}^{x}\right|\right\} \text { and } \max \left\{\left|\Delta_{i, j}^{y}\right|\right\},
$$

where $\Delta_{i, j}^{x}$ and $\Delta_{i, j}^{y}$ are the partial derivatives of the phase and the maxima is evaluated in the $k \times k$ neighbourhood of the given pixel. As in phase derivative variance map, this value has to be negated to represent goodness.

\section{Domain masking}

Before unwrapping the model, the domain of the model to be unwrapped has to be segmented. The regions outside the domain are masked out thereby making the algorithm to work within the chosen model domain. One can consider mask as a sort of quality map whose pixels take the value of zero. The zero-valued pixels indicate the lowest quality phase values that are to be masked out or ignored. Use of such an approach in identifying the model regions with cut-outs is envisaged in this paper.

A domain masking file in the form of binary image is initially made such that the unwanted regions are given black colour whereas domain of interest is given white colour. In this work the standard package of Adobe Photoshop is used for making the binary image in aiding domain masking. The branch-cut/quality-guided path follower algorithm initially reads this file and then completely masks the unwanted region. Thus, the phase unwrapping is carried out in the region of interest while the unwanted region is totally omitted.

\section{Implementation of autonomous algorithms}

The quality-guided path follower algorithm for unwrapping and the flood-fill procedure of Goldstein's branch-cut use complicated unwrapping paths. Quality-guided unwrapping algorithms use quality map whereas branch-cut guides unwrapping path based upon avoiding branch-cut pixels. In the implementation of these algorithms, a lot of book keeping is essential. Each pixel present in the phase map is assigned a bitflag which are array of bytes whose lowest order bits store the first flags and so on. In the case of branch-cut algorithm, seven flags are used which represent positive residue, negative residue, branch-cut, image border (domain masking), balanced residue, active residue and unwrapped pixel. At first, the boundary flag is set based upon the binary image. The balanced residue keeps track of which residues have been balanced with branch-cuts. The active residue flag indicates whether a residue is active, which means it is connected to the current set of branch-cuts. Any residue encountered during the search of the box is marked active. The algorithm maintains a list of pixel indices for 


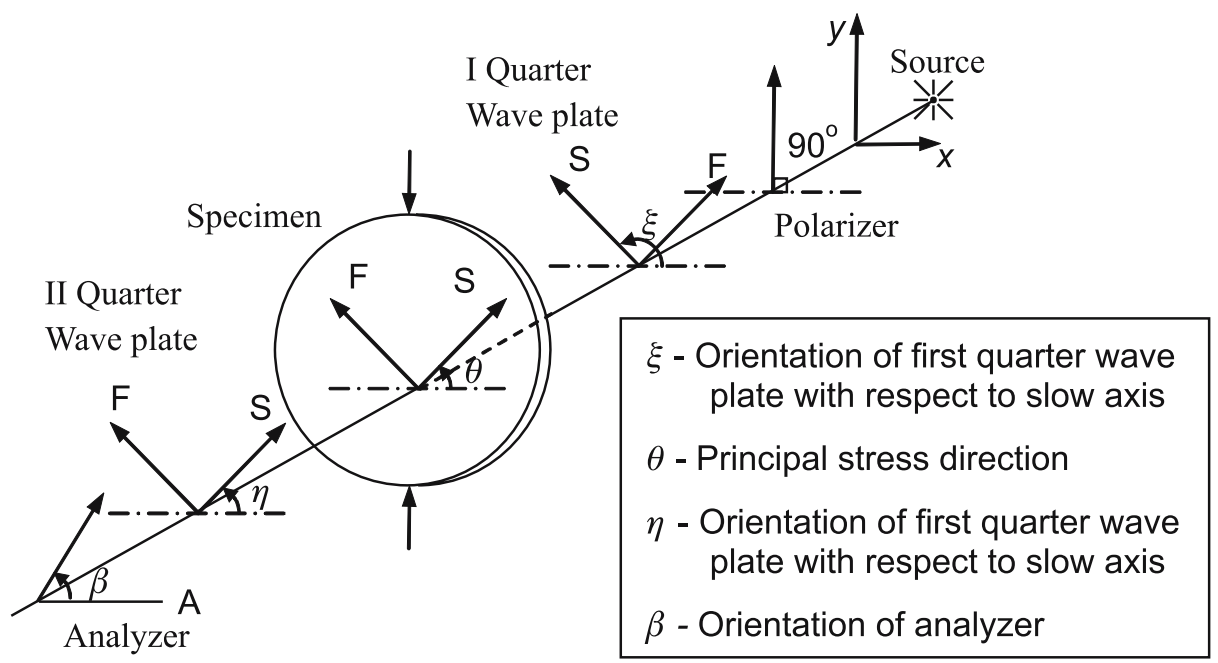

Figure 2. Generic arrangement of a circular polariscope.

keeping track of which pixels adjoin the unwrapped pixels. This list is referred to as adjoin list. The algorithm unwraps in an iterative fashion by selecting and removing a pixel from the adjoin list and inserting four neighbours in the list avoiding branch-cut and the pixels that are already unwrapped. This process continues until the adjoin list becomes empty. The algorithm stores the result in a solution array.

The quality-guided path follower algorithm uses a separate array to store the information of quality map. The quality information is added to the adjoin list and all the pixels are graded from the highest to lowest in the descending order. Every time, the unwrapping algorithm checks with this list and performs unwrapping operation based on quality value. The result is stored in a solution array as before.

\section{Performance of the autonomous unwrapping algorithms for a benchmark photoe- lastic problem}

In this paper, unwrapping of a benchmark problem in digital photoelasticity is done by branchcut algorithm as well as based on three quality maps. The performance is quantitatively compared for identifying the best quality map applicable to digital photoelastic analysis. Problem of a disk under diametral compression (dia $=60.14 \mathrm{~mm}$, thickness $=5.1 \mathrm{~mm}$, Fsigma $=11.23 \mathrm{~N} / \mathrm{mm} /$ fringe, load $=820$ hbox $N$ ) is chosen. The experimental data of isochromatic data is extracted using a six-step PST algorithm based on the circular polariscope arrangement (figure 2). The optical arrangements used for six-step PST are shown in table 1 (Ajovalasit et al 1998). From the intensity equations, the isoclinic and isochromatic parameter are obtained as Ramesh (2000)

$$
\begin{aligned}
& \theta_{c}=\frac{1}{2} \tan ^{-1}\left(\frac{I_{5}-I_{3}}{I_{4}-I_{6}}\right)=\frac{1}{2} \tan ^{-1}\left(\frac{I_{a} \sin \delta \sin 2 \theta}{I_{a} \sin \delta \cos 2 \theta}\right) \\
& \delta_{c}=\tan ^{-1}\left(\frac{\left(I_{5}-I_{3}\right) \sin 2 \theta_{c}+\left(I_{4}-I_{6}\right) \cos 2 \theta_{c}}{\left(I_{1}-I_{2}\right)}\right)=\tan ^{-1}\left(\frac{I_{a} \sin \delta_{c}}{I_{a} \cos \delta_{c}}\right),
\end{aligned}
$$


Table 1. Optical arrangements for six-step phase shifting technique.

\begin{tabular}{lccc}
\hline$\xi$ & $\eta$ & $\beta$ & Intensity \\
\hline $3 \pi / 4$ & $\pi / 4$ & $\pi / 2$ & $I_{1}=I_{b}+\frac{I_{a}}{2}(1+\cos \delta)$ \\
$3 \pi / 4$ & $\pi / 4$ & 0 & $I_{2}=I_{b}+\frac{I_{a}}{2}(1-\cos \delta)$ \\
$3 \pi / 4$ & 0 & 0 & $I_{3}=I_{b}+\frac{I_{a}}{2}(1-\sin 2 \theta \sin \delta)$ \\
$3 \pi / 4$ & $\pi / 4$ & $\pi / 4$ & $I_{4}=I_{b}+\frac{I_{a}}{2}(1+\cos 2 \theta \sin \delta)$ \\
$\pi / 4$ & 0 & 0 & $I_{5}=I_{b}+\frac{I_{a}}{2}(1+\sin 2 \theta \sin \delta)$ \\
$\pi / 4$ & $3 \pi / 4$ & $\pi / 4$ & $I_{6}=I_{b}+\frac{I_{a}}{2}(1+\cos 2 \theta \sin \delta)$ \\
\hline
\end{tabular}

where $I_{a}$ is the light intensity accounting for the amplitude of light, $\theta$ is the orientation of the principal stress direction with respect to $x$-axis and $\delta$ is the fractional retardation. In (7) and (8), the subscript $c$ indicates that the principal values of the inverse trigonometric functions are referred. Ramesh (2000) recommends that $\theta_{c}$ is to be evaluated by atan () function and $\delta_{c}$ is to be evaluated by atan2 () function. The phase shifted images are experimentally recorded with a monochromatic light source (Sodium vapour, $\lambda=589.3 \mathrm{~nm}$ ) and the intensity of light transmitted is recorded by a monochrome CCD camera (TM-560 PULNiX) having a resolution of $512 \times 512$ pixels.

Using (7) and (8), the fractional retardation for the problem of a circular disk under diametral compression is obtained from the experimentally generated phase shifted images. The phase map is obtained using the following relations Ramesh (2000)

$$
\begin{gathered}
\delta_{p}= \begin{cases}\delta_{c} & \text { for } \quad \delta_{c}>0 \\
2 \pi+\delta_{c} & \text { for } \quad \delta_{c} \leq 0\end{cases} \\
g(x, y)=\frac{255}{2 \pi} \delta_{p},
\end{gathered}
$$

where $g(x, y)$ is the gray level of the pixel at the location $(x, y)$. The phase map thus obtained is shown in figure $3 \mathrm{a}$. Near the loading points the phase map is discontinuous, which is due

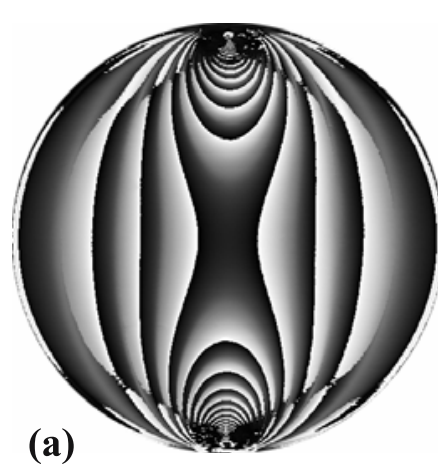

(a)

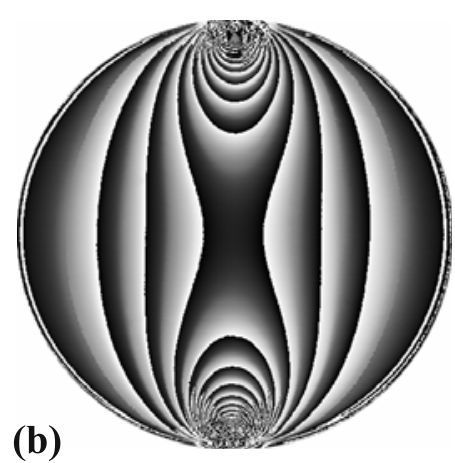

(b)

Figure 3. Phase map obtained for the problem of a disk under diametral compression using six-step algorithm (a) phase map with ambiguity (directly obtained from experiment), (b) phase map after correcting ambiguity using interactive approach. 


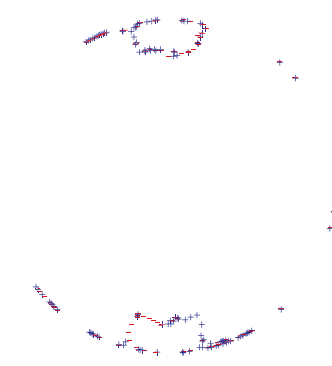

(a) (b)

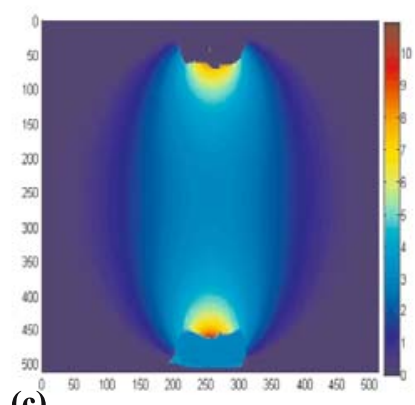

(c)

Figure 4. Goldstein's approach to the problem of disk under a diametral compression (a) residues, (b) branch-cuts, (c) Matlab plot of unwrapped phase obtained.

to the ambiguity in the sign of the fractional retardation. For unwrapping to be effective, the phase map should be free of ambiguity. The phase map is corrected using interactive approach (Ramesh \& Mangal 1999). The resulting phase map (figure 3b) is unwrapped by various autonomous algorithms mentioned previously to assess their performance.

\section{Qualitative analysis}

\subsection{Goldstein's branch-cut algorithm}

The domain masking concept is applied here and the region outside the disk is completely masked. The phase map gray scale intensity values (0-255) are scaled down to $0-1$ and stored in an array. This array is then checked for residues by employing the $2 \times 2$ path inconsistency test. Wherever there are inconsistencies, the corresponding locations are marked as residues. Figure $4 \mathrm{a}$ shows the residues obtained by this algorithm. The positive residues are represented as plus symbol and the negative ones as minus symbol. Residues occupy just one pixel and to appreciate the nature of residues globally and to assess their presence visually, each residue location is shown by plotting a $4 \times 4$ array surrounding the residue pixel. Now these residues are suitably connected to provide the necessary branch-cuts. Goldstein's algorithm is designed to minimise the lengths of the branch-cuts by connecting the residues using nearest neighbour approach. Figure $4 \mathrm{~b}$ shows the branch-cuts generated by this algorithm on the phase map around the loading point region. The phase map is then unwrapped using a flood-fill algorithm. Seed point is selected within the model domain by the user and integral value of the fringe order is given. The algorithm unwraps the entire phase map autonomously by unwrapping the non-branch-cut pixels first followed by branch-cut pixels. Figure $4 \mathrm{c}$ shows the Matlab plot of the unwrapped phase distribution. The algorithm has unwrapped over the entire domain except at the loading zones where it has performed poorly.

\subsection{Quality-guided path follower algorithm}

Figures $5 \mathrm{a}-\mathrm{c}$ show the quality map for the disk under diametral compression by pseudocorrelation, phase derivative variance and maximum phase gradient approach. In the quality map, the black areas are of low quality and the white areas are of high quality. Figures $5 \mathrm{~d}-\mathrm{f}$ show the Matlab plot of unwrapped phase distribution. All the algorithms have performed well over the entire domain except at regions closer to the loading point. 


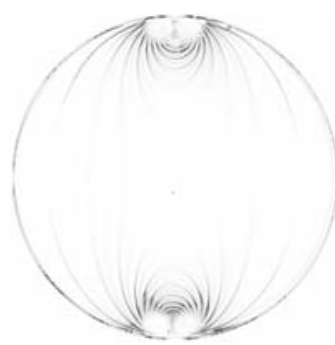

(a)

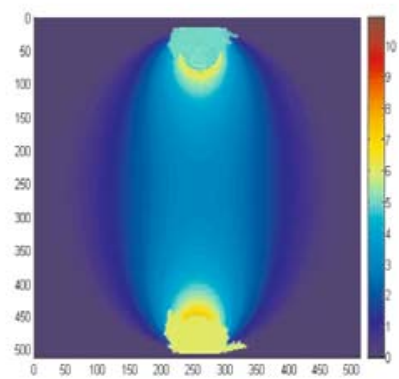

(d)

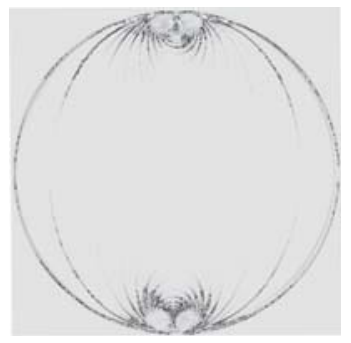

(b)

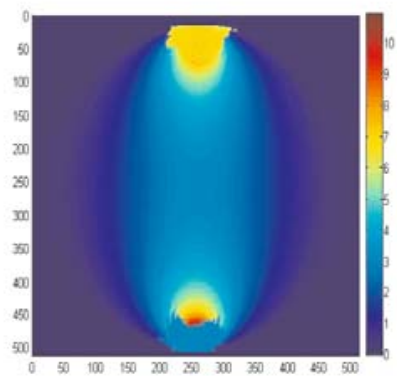

(e)

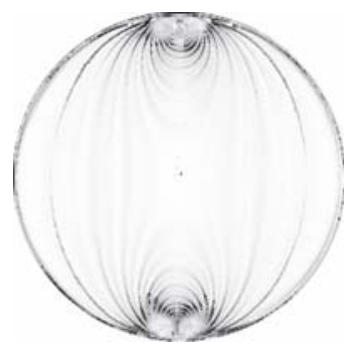

(c)

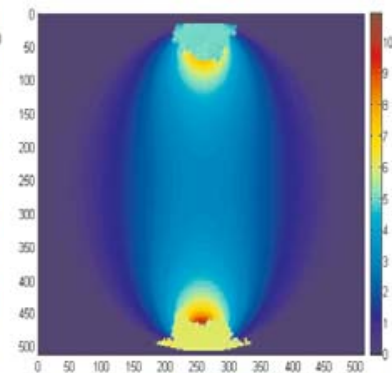

(f)

Figure 5. Quality maps and unwrapped phase for the problem of disk under a diametral compression by various schemes (a) pseudo-correlation, (b) phase derivative variance, (c) maximum phase gradient, (d-f) Matlab plot of unwrapped phase obtained using respective quality maps.

\section{Quantitative analysis}

Figure 6 shows the variation of fringe order obtained for the problem of a disk under diametral compression for the line $y / R=0.5$ by the methods of branch-cut, pseudo-correlation, phase derivative variance, maximum phase gradient compared with theory. The comparison is quite

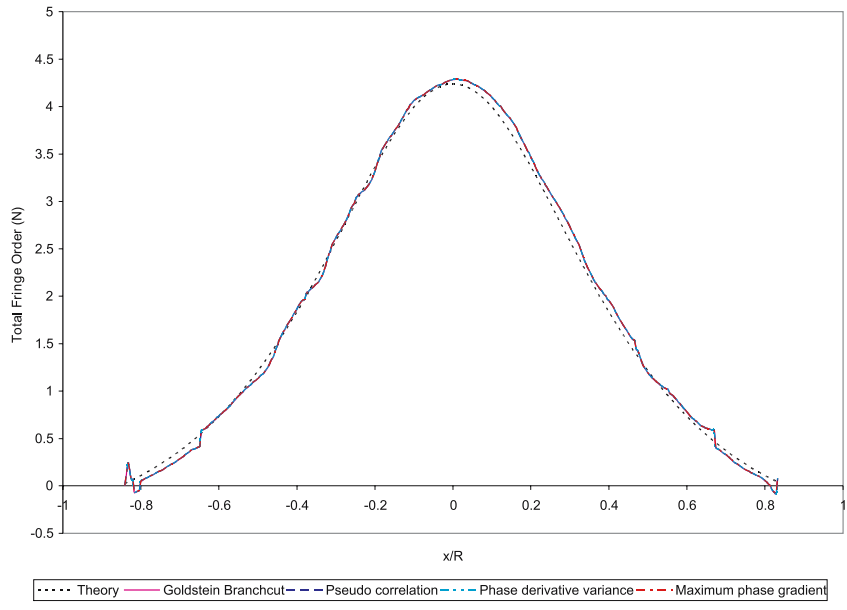

Figure 6. Experimentally obtained delta with theoretical delta for the problem of disk under diametral compression line at the line $y / R=0.5$ by various autonomous phase algorithms: Goldstein's branchcut, quality-guided algorithms (pseudo-correlation, phase derivative variance and maximum phase gradient) and theory. 


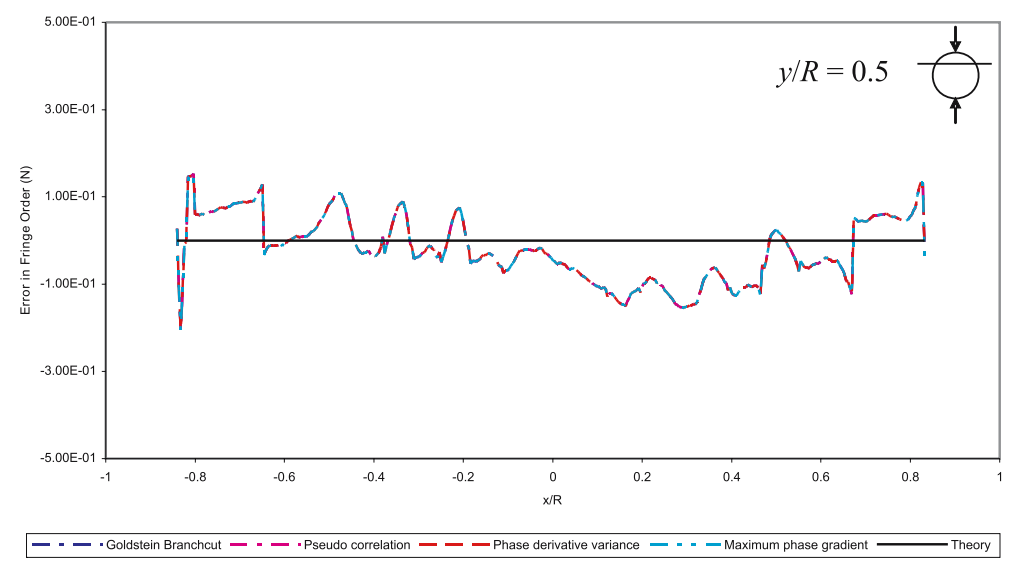

Figure 7. Error plot of experimentally obtained delta for the problem of disk under diametral compression line at the line $y / R=0.5$ by various autonomous phase algorithms: Goldstein branch-cut, quality-guided algorithms (pseudo-correlation, phase derivative variance and maximum phase gradient).

good. Figure 7 shows the error in fringe order along the line $y / R=0.5$ for all the algorithms. The average absolute error in fringe order by various algorithms is only 0.063 fringe order. A maximum error of $+0 \cdot 15 /-0.2$ fringe order is obtained at the edges which are due to the time edge effect developed in the specimen. The total percentage area covered by different algorithms is shown in table 2 . Phase derivative variance covers a maximum area of $96.1 \%$ whereas pseudo-correlation covers the least area of $94.8 \%$. Table 2 further gives the computational time (Intel P-IV, $3.4 \mathrm{MHz}$ processor) taken by different algorithms. All the algorithms take less than a second for the unwrapping process. In an overall sense, the algorithm of phase derivative variance is better than that of the other algorithms.

\section{Performance of the autonomous algorithms for a stress frozen slice with cut-outs}

The experimental phase shifted images of a stress frozen slice are recorded by using a monochromatic CCD camera (DC-700 SONY) having a spatial resolution of $768 \times 576$ pixels. Using the six phase shifted images of the slice, the fractional retardation for the slice is

Table 2. Quantitative description of the performance of various autonomous algorithms: Branch-cut, pseudo-correlation, phase derivative variance, maximum phase derivative.

\begin{tabular}{|c|c|c|c|}
\hline Algorithm & $\begin{array}{c}\text { Area covered }(\%) \\
\text { Image size }(512 \times 512)\end{array}$ & Time (sec) & $\begin{array}{l}\text { Accuracy of data along } \\
\text { the line } y / R=0 \cdot 5 \\
\text { (average modulus error } \\
\text { in fringe order) }\end{array}$ \\
\hline Branch cut & $95 \cdot 2$ & $<1$ & 0.063 \\
\hline Pseudo-correlation & $94 \cdot 8$ & $<1$ & 0.063 \\
\hline Phase derivative variance & $96 \cdot 1$ & $<1$ & 0.063 \\
\hline Maximum phase derivative & 95.7 & $<1$ & 0.063 \\
\hline
\end{tabular}



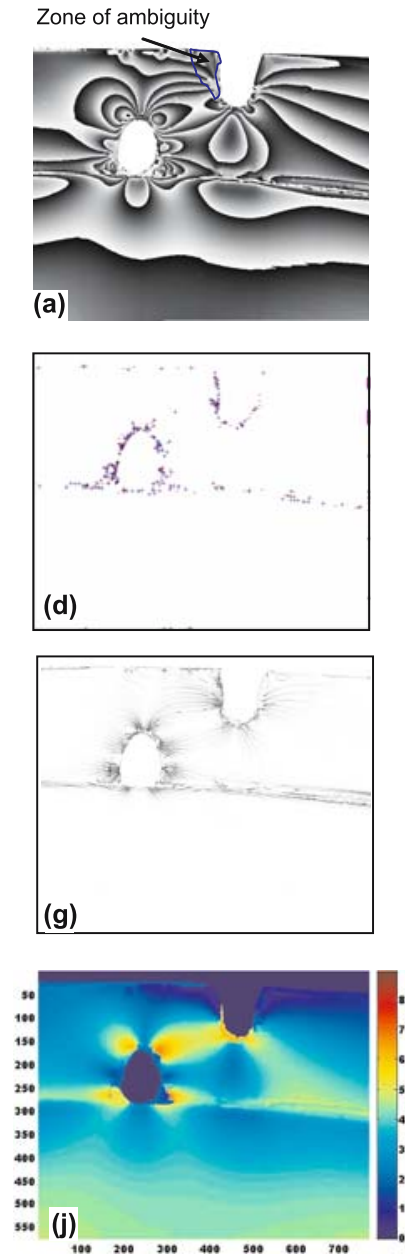
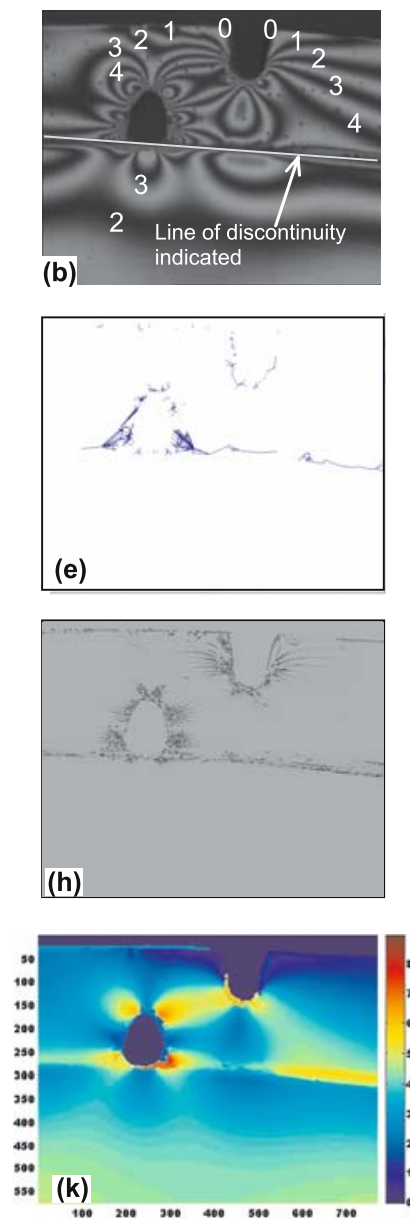
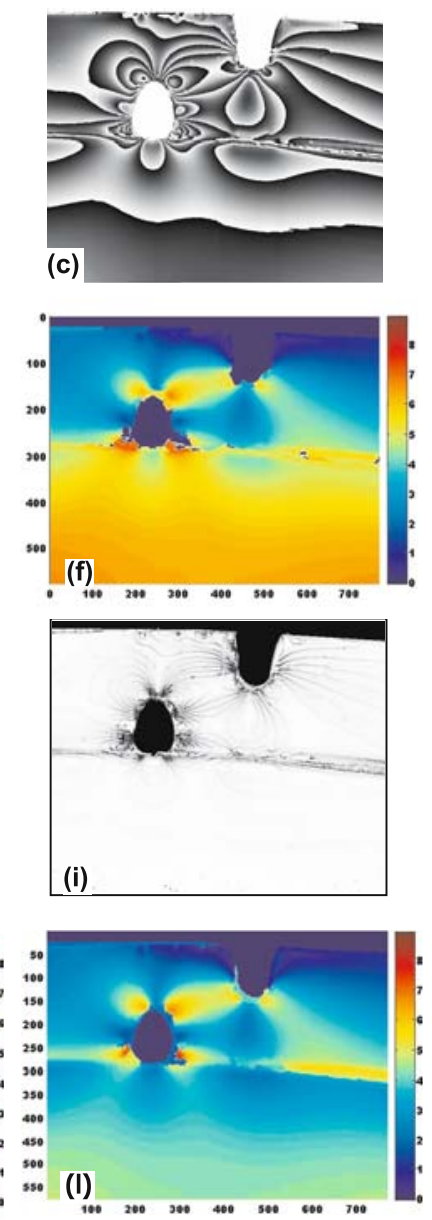

Figure 8. Goldstein's approach, quality maps and unwrapped phase for the stress frozen slice by various schemes when the seed point is selected on the top zone (a) phase map with ambiguity, the background is cleaned for clarity, (b) dark field circular polariscope image in monochrome source shown along with fringe ordering, (c) corrected phase map after ambiguity removal, (d-f) residues, branch-cut and unwrapped plot, (g) pseudo-correlation, (h) phase derivative variance, (i) maximum phase gradient quality maps, (j-l) Matlab plot of unwrapped phase obtained using quality-guided algorithm.

calculated invoking (7) and (8). The resulting phase map is shown in figure 8a. Figure 8b shows the dark field image obtained using a monochromatic light source. The fringe order is marked on the figure $8 \mathrm{~b}$ for easy understanding. Figure $8 \mathrm{~b}$ clearly shows that there is a physical discontinuity in the stress frozen slice and two zones can be identified viz. the top and bottom zones. The phase map obtained is of a distinct nature in the respective zones (figure $8 \mathrm{c}$ ). In the bottom zone, the fractional fringe order decreases in the direction of increase in total fringe order whereas in the top zone fractional fringe order increases in the direction of increase in total fringe order. In the top zone, ambiguous region is closer to the open cutout and this is corrected by interactive approach. It is to be noted that only the top zone is corrected for ambiguity whereas the bottom zone is untouched. 


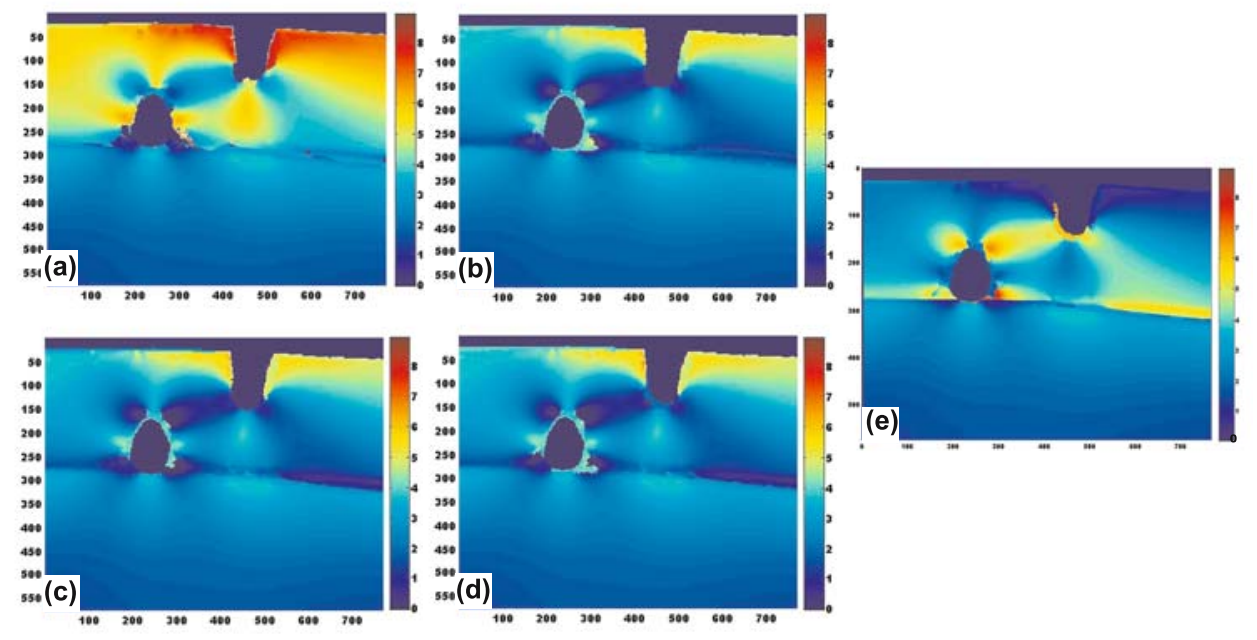

Figure 9. Matlab plot of Unwrapped phase for stress frozen slice by various schemes when the seed point is selected on the bottom zone (a) branch-cut, (b) pseudo-correlation, (c) phase derivative variance, (d) maximum phase gradient, (e) by domain delimiting using simple scanning procedure (Madhu \& Ramesh 2007).

Figure 8d shows the residues obtained by Goldstein's approach and the branch-cuts are shown in figure $8 \mathrm{e}$. The unwrapping is done by selecting a seed point from the top zone. The unwrapped phase map is shown in figure $8 \mathrm{f}$. Figures $8 \mathrm{~g}-1$ show the quality map and the unwrapped phase for the stress frozen slice by pseudo-correlation, phase derivative variance and maximum phase gradient approach. For each of these cases, seed point for unwrapping is selected in the top zone. All the algorithms have given a smooth variation of total fringe order and are also similar in performance visually. Figures $9 \mathrm{a}-\mathrm{d}$ show Matlab plot of unwrapped phase map when the seed point is selected in the bottom zone. Figure $9 \mathrm{e}$ shows the unwrapping by domain delimiting using a simple scanning approach (Madhu \& Ramesh 2007). Here, the phase map is appropriately segmented as simply connected objects thereby avoiding the cut-outs. In figures $8 \mathrm{f}$ and $8 \mathrm{j}-1$ using a seed point in the top zone, the unwrapping of the top zone compares well with figure $9 \mathrm{e}$. While using the seed point from the bottom zone (figures 9a-d), the bottom zone compares well with figure 9e. Thus, the autonomous phase unwrapping methodologies can be erroneous if the phase map has distinct zones of fractional retardation variation. Either the ambiguity removal algorithm must take care of this aspect or one should use a domain delimiting approach in conjunction with phase unwrapping.

However, due to domain masking even in the presence of cut-outs, both the Goldstein's branch-cut and quality-guided path follower unwrapping algorithms are capable of unwrapping for the whole model domain using complicated unwrapping paths. The importance of domain masking can be appreciated if one performs unwrapping without domain masking. Figure 10 shows the Matlab plot of unwrapped phase obtained without domain masking of cut-outs using the algorithms of branch-cut and phase derivative variance. Unwrapping is done inside the cut-outs too and this leads to an error in fringe order around the cut-out region. The zoomed up portion of the Matlab plot of the unwrapped phase near one of the cut-outs is shown in figure 11 . One could clearly see poor unwrapping around the cut-out while using branch-cut whereas phase derivative variance has faired well. Figure 12 shows the total fringe order variation along line $\mathrm{AB}(y / H=0.04)$ marked on figure 11a. In the case of phase 

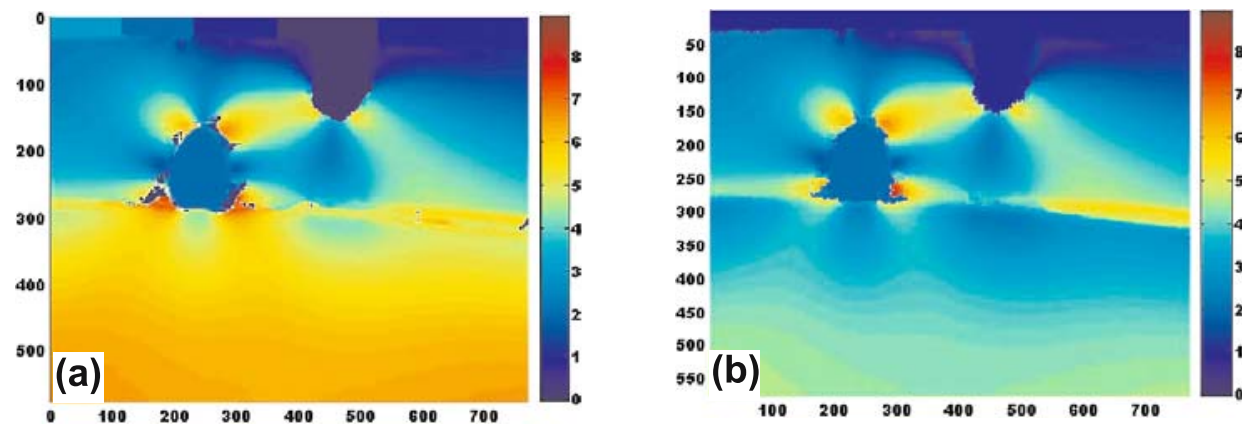

Figure 10. Matlab plot of unwrapped phase for stress frozen slice by various schemes when the seed point is selected on the top zone without domain masking (a) branch-cut, (b) phase derivative variance.

derivative variance with domain masking, the fringe order is zero in the cut-out region. The fringe order is around five on the left side of the cut-out whereas it is around seven on the right side. If domain masking is not done, fringe order in the cut-out region is wrongly estimated as 1.98. This error has propagated to both sides of the cut-out and the fringe order is under estimated. Hence, domain masking is essential. In the case of branch-cut algorithm with domain masking, the fringe order in the cut-out region is zero. On the right side of the cut-out the estimated fringe order matches with phase derivative variance but it is over estimated on
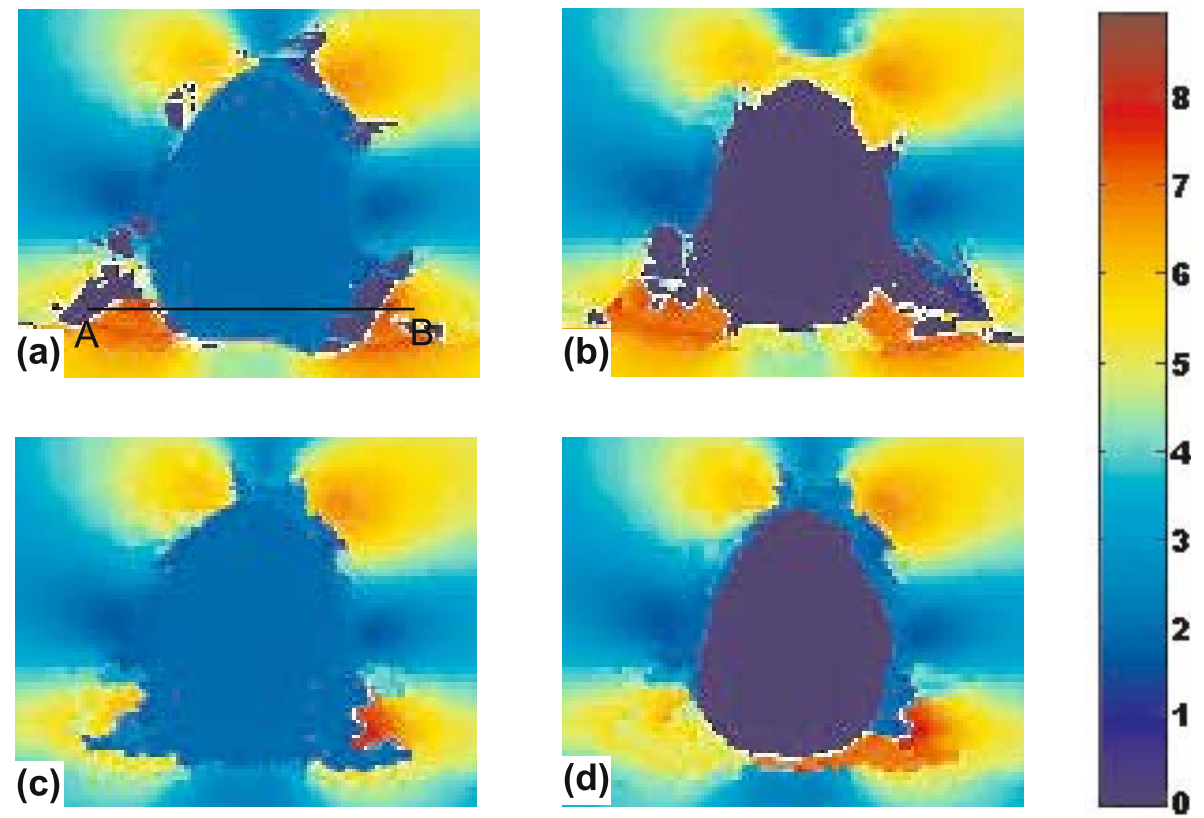

Figure 11. Zoomed portion near the cut-out of the Matlab plot of unwrapped phase for stress frozen slice by various schemes when the seed point is selected on the top zone (a) branch-cut without domain masking (figure 10a), (b) branch-cut with domain masking (figure 8f), (c) phase derivative variance without domain masking (figure 10b), (d) phase derivative variance with domain masking (figure 8k). 


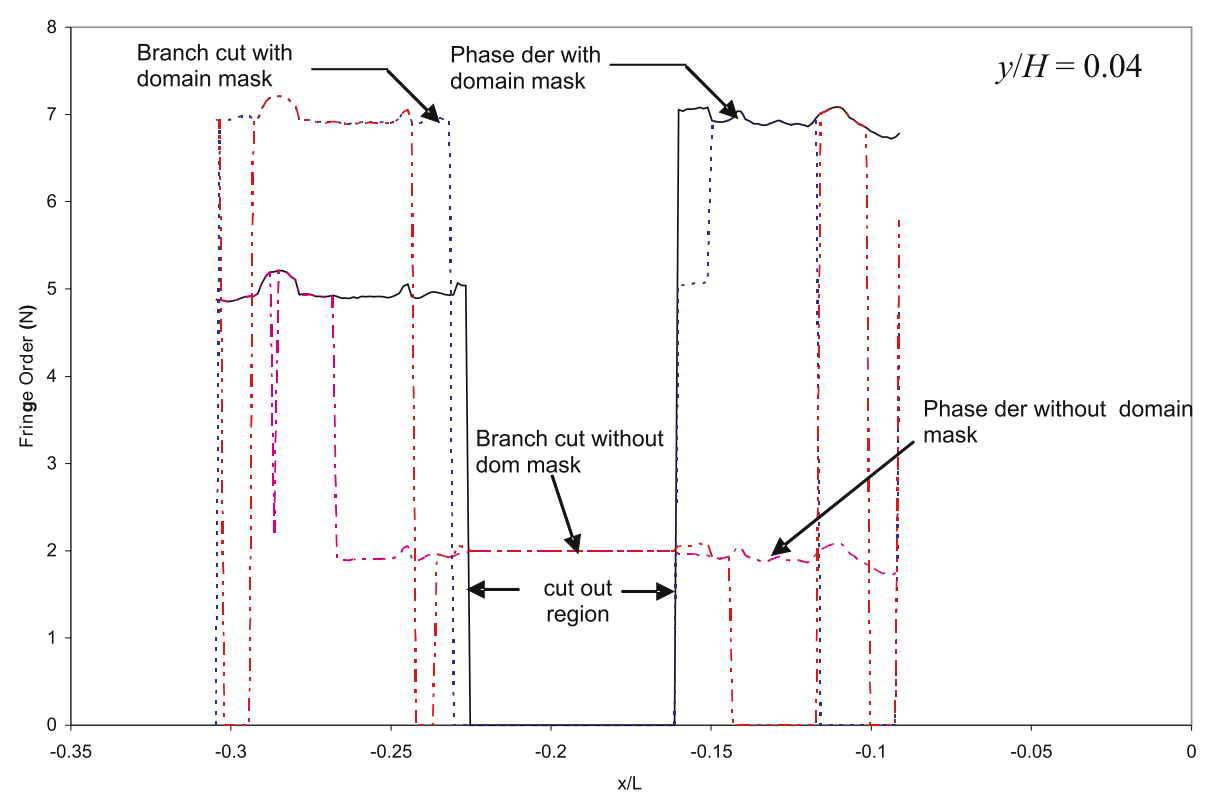

Figure 12. Comparison of total fringe order variation along line $\mathrm{AB}(y / H=0.04)$ as shown in figure 11a obtained using the algorithms of phase derivative variance and branch-cut algorithm (without and with domain masking).

the left side of the cut-out. Without domain masking it has estimated wrongly in the cut-out region and it also over estimates the fringe order on the left side of the cut-out. Thus, branchcut algorithm (with and without domain masking) has not performed well when there is a cut-out.

\section{Conclusion}

Goldstein's branch-cut approach and quality-guided phase unwrapping methodologies are useful for autonomous phase unwrapping even in the presence of cut-outs. In the case of simply connected models, the quality-guided algorithms and Goldstein's branch-cut algorithm are similar in their performances and compare well with analytical solution. However, all these algorithms perform poorly nearing the loading points due to poor spatial resolution. For effective unwrapping in such zones methodology of optically enhanced tiling is recommended (Ramesh \& Sreedhar 1998). In complex models with cut-outs domain masking is essential. In areas surrounding the cut-out, quality-guided algorithms are far superior in performance than the branch-cut approach. Among the quality-guided algorithms, phase derivative variance and maximum phase gradient are recommended for their accuracy and higher domain area coverage. Although quality-guided unwrapping algorithms work well for models having uniform fringe gradients, when it comes to problems with physical discontinuity having clearly demarcated zones of different fractional fringe order gradients, the algorithms fail. The combined use of domain delimiting followed by quality-guided phase unwrapping within each region would be more effective in such cases. This would involve only a minimal user intervention which in many cases is desirable (Ashokan et al 2006). 


\section{References}

Ajovalasit A, Barone S, Petrucci G 1998 A method for reducing the influence of the quarter wave plate error in phase shifting photoelasticity. J. Strain Analysis for Eng. Des. 33(3): 207-216

Ashokan K, Ramji M, RajaGuruprasath R, Ramesh K 2006 Autonomous isochromatic phase unwrapping by domain delimiting in digital photoelasticity. CD Proc. VII Int. Conf. on Optoelectronics Fibre Optics and Photonics (Photonics 2006), December 13-16, University of Hyderabad, Hyderabad, India

Asundi A K, Wensen Z 1998 Fast phase-unwrapping algorithm based on a gray-scale mask and floodfill. Appl. Opt. 37(23): 5416-5420

Bone D J 1991 Fourier fringe analysis: Two-dimensional phase unwrapping problem. Appl. Opt. 30(25): 3627-3632

Ekman M J, Nurse A D 1998 Absolute determination of the isochromatic parameter by load-stepping photoelasticity. Exp. Mech. 38(3): 189-195

Ghiglia D C, Mastin G A, Romero L A 1987 Cellular-automata method for phase unwrapping. J. Opt. Soc. Am. 4(1): 267-280

Ghiglia D C and Pritt M D 1998 Two-dimensional phase unwrapping: Theory, algorithms and software. (US: Wiley-InterScience)

Goldstein R M, Zebker H A, Werner C L 1988 Satellite radar interferometry: two-dimensional phase unwrapping. RadioScience 23(4): 713-720

Huntley J M 1989 Noise-immune phase unwrapping algorithm. Appl. Opt. 28(15): 3268-3270

Itoh 1982 Analysis of phase unwrapping problem. Appl. Opt. 21(14): 2470

Jones I A, Wang P 2003 Complete fringe order determination in digital photoelasticity using fringe combination matching. Strain 39(3): 121-130

Lim H, Xu W, Huang X 1995 Two new practical methods for phase unwrapping. Proc. International Geoscience and Remote Sensing Symposium, Tokyo, Japan, August 18-21, IEEE, Piscataway, NJ, 196-198

Madhu K R, Ramesh K 2007 New boundary information encoding and auto seeding for effective phase unwrapping of specimens with cut-outs. Strain 43: 54-57

Prashant P L, Madhu K R, Ramesh K 2004 New initiatives in phase unwrapping in digital photoelasticity. Proc. Int. Conference on Experimental Mechanics, Nov 29 - Dec 1, Singapore

Quiroga J A, Gonzalez-Cano A 2000 Separation of isoclinics and isochromatics from photoelastic data with a regularised phase-tracking technique. Appl. Opt. 39(17): 2931-2940

Quiroga J A, Gonzalez-Cano A, Bernabue E 1995 Phase unwrapping algorithm based on adaptive criterion. Appl. Opt. 34(14): 2560-2563

Ramesh K 2000 Digital Photoelasticity: Advanced techniques and applications. (Berlin: SpringerVerlag)

Ramesh K, Mangal S K 1999 Phase-shifting calculations in 2-D photoelasticity: revisited. J. Aeronautical Soc. India 52(2): 121-136

Ramesh K, Sreedhar K 1998 Optically enhanced tiling (OET) in digital fringe pattern analysis. Strain 34(4): 133-134

Sai Prasad V, Khode R K, Madhu K R, Ramesh K 2002 Phase unwrapping in digital photoelasticity by tiling. Proc. of Int. Symposium on Experimental Mechanics, Dec 28-30, Taipei, Taiwan

Sai Prasad V, Madhu K R, Ramesh K 2004 Towards effective phase unwrapping in digital photoelasticity. Optics and Lasers in Eng. 42: 421-436

Sai Prasad V, Ramesh K 2003 Background intensity effects on various phase shifting techniques in photoelasticity. Proc. Conference on Optics and Photonics in Engineering, Jan 6-8, NSIT, New Delhi

Servin M, Quiroga J A 2001 Isochromatics demodulation from a single image using the regularised phase tracking technique. J. Modern Opt. 48(3): 521-531

Siegmann P, Backman D, Patterson E A 2005 A robust approach to demodulating and unwrapping phase-stepped photoelastic data. Exp. Mech. 45(3): 278-289 
Wang Z F and Patterson E A 1995 Use of phase stepping with demodulation and fuzzy sets for birefringence measurement. Optics and Lasers in Eng. 22: 91-104

$\mathrm{Xu} \mathrm{W}$ and Cumming I 1996 A region growing algorithm for InSAR phase unwrapping, Proc. International Geoscience and Remote Sensing Symposium, Lincoln, NE, May 27-31, IEEE, Piscataway, NJ, 2044-2046 